\title{
SHORT DYNAMIC FIBRILS IN SUNSPOT CHROMOSPHERES
}

\author{
L. ROUPPE VAN DER VOORT ${ }^{1}$ AND J. DE LA CRUZ RodríGueZ ${ }^{2}$ \\ ${ }^{1}$ Institute of Theoretical Astrophysics, University of Oslo, P.O. Box 1029 Blindern, N-0315 Oslo, Norway \\ 2 Department of Physics and Astronomy, Uppsala University, Box 516, SE-75120 Uppsala, Sweden \\ Received 2013 June 25; accepted 2013 August 18; published 2013 September 25
}

\begin{abstract}
Sunspot chromospheres display vigorous oscillatory signatures when observed using chromospheric diagnostics such as the strong $\mathrm{Ca}$ II lines and $\mathrm{H} \alpha$. New high-resolution sunspot observations from the Swedish $1 \mathrm{~m}$ Solar Telescope show the ubiquitous presence of small-scale, periodic, jet-like features that move up and down. This phenomenon has not been described before. The typical width of these features is about 0.3 and they display clear parabolic trajectories in space-time diagrams. The maximum extension of the top of the jets is lowest in the umbra, a few $100 \mathrm{~km}$, and progressively longer further away from the umbra in the penumbra, with the longest extending more than $1000 \mathrm{~km}$. These jets resemble the dynamic fibrils found in plage regions but at smaller extensions. Local thermodynamic equilibrium inversion of spectropolarimetric Ca II 8542 observations enabled a comparison of the magnetic field inclination and properties of these short jets. We find that the most extended of these jets also have longer periods and tend to be located in regions with more horizontal magnetic fields. These results are direct observational confirmation of the mechanism of long-period waves propagating along inclined magnetic fields into the solar chromosphere. This mechanism was identified earlier as the driver of dynamic fibrils in plage, part of the mottles in the quiet Sun, and the type I spicules at the limb. The sunspot dynamic fibrils that we report here represent a new class of manifestation of this mechanism, distinct from the transient penumbral and umbral micro-jets reported earlier.
\end{abstract}

Key words: Sun: atmosphere - Sun: chromosphere - Sun: oscillations - sunspots

Online-only material: animations, color figures

\section{INTRODUCTION}

The solar chromosphere is dominated by linear structures that display vigorous dynamical evolution on time scales ranging from seconds to minutes. Off the limb, elongated features are observed as spicules with typical linear dimensions between 5 and $10 \mathrm{Mm}$ (for a recent review, see Tsiropoula et al. 2012). High-resolution Hinode observations revealed the existence of at least two types of spicules (De Pontieu et al. 2007b), of which the second class, or type II spicules, was found to be most abundant in the solar atmosphere. Type I spicules are mostly found in active regions (ARs), are the minority species in the quiet Sun, and are virtually absent in coronal holes (Pereira et al. 2012). They are characterized by clear rising and descending phases on time scales of 140-400 s and their tops describe a distinct parabolic trajectory in the space-time domain. Their properties agree well with what was measured on the solar disk for dynamic fibrils in AR plage by De Pontieu et al. (2007a) and for some mottles in the quiet Sun by Rouppe van der Voort et al. (2007).

In strong plage regions, dynamic fibrils display a striking quasi-periodic up and down motion, with dynamic fibrils reoccurring at roughly the same location over practically the full duration of the observational datasets. Often, dynamic fibrils show group behavior with neighboring structures moving in harmony, or with a small phase delay. Numerical simulations demonstrated that this wave-like behavior is driven by slowmode magneto-acoustic shocks that result from $p$-mode waves leaking from the photosphere into higher atmospheric layers (Suematsu et al. 1982; Shibata \& Suematsu 1982; Hansteen et al. 2006; De Pontieu et al. 2007a; Heggland et al. 2007, 2011). De Pontieu et al. (2004) showed that the five minute $p$-mode oscillations that earlier were considered to be evanescent above the photosphere can propagate progressively more efficiently along increasingly more horizontal magnetic fields. When these long-period waves propagate to higher atmospheric regions with lower density, they turn into shocks that drive the observed dynamic fibrils. Such a change in the cutoff frequency for wave propagation along slanted fields had earlier been described by Michalitsanos (1973) and Bel \& Leroy (1977) but was not appreciated in the subsequent literature, except for Suematsu (1990).

In this paper, we study comparable phenomena in sunspots. Sunspot atmospheres are also clearly dominated by waves and shocks: chromospheric diagnostics like $\mathrm{H} \alpha$ and the strong $\mathrm{Ca}$ II lines show sunspots and pores oscillating in intensity and Doppler signal coherently over large fractions of their surface areas. This captivating phenomenon is particularly striking in the $\mathrm{Ca}$ II $\mathrm{H}$ and $\mathrm{K}$ lines, where the oscillations are manifested as strong intensity variations or umbral flashes and running penumbral waves (see, e.g., the movies accompanying Rouppe van der Voort et al. 2003 and Nagashima et al. 2007). There is observational evidence that waves in the chromospheres of sunspots are driven from below (see, e.g., the review by Lites 1992). Bard \& Carlsson (2010) perform non-local thermodynamic equilibrium (LTE) radiation hydrodynamic simulations of the propagation of acoustic waves in sunspot umbrae. These authors conclude that the $\mathrm{Ca}$ II $\mathrm{H}$ and $\mathrm{K}$ umbral flashes result from increased emission of the local solar material during the passage of waves originating in the photosphere and steepening to shocks in the chromosphere. While umbral flashes are a relatively large-scale phenomenon with emission patches several arcseconds across, there have been reports that these features harbor fine structure, as was concluded from polarimetric inversions (Socas-Navarro et al. 2000a, 2000b) and direct imaging (Socas-Navarro et al. 2009; Henriques \& Kiselman 2013). In this paper, we present high-resolution $\mathrm{H} \alpha$ and $\mathrm{Ca}$ II 8542 observations that reveal for 

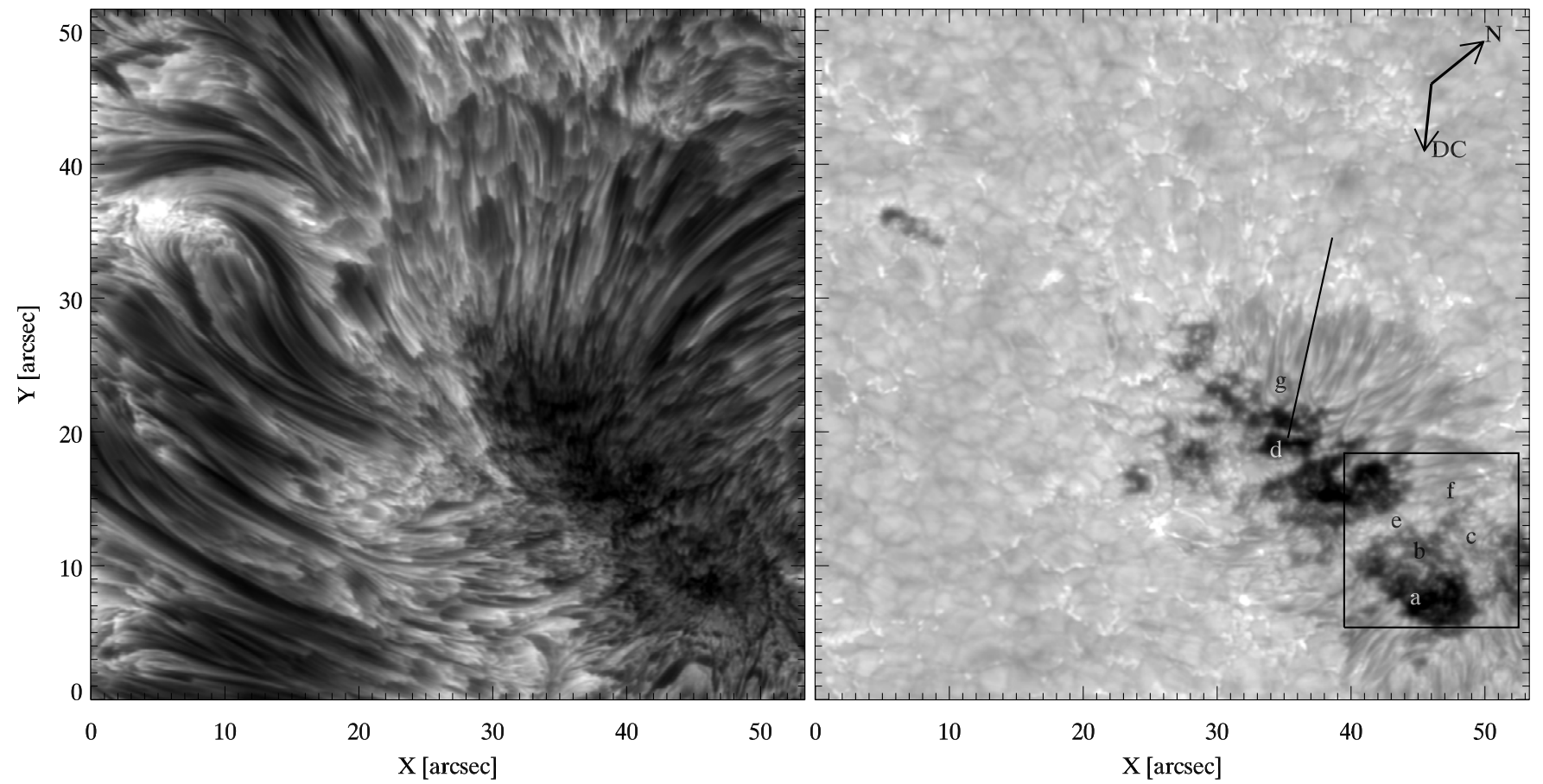

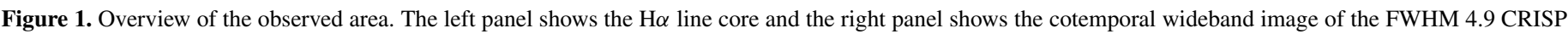

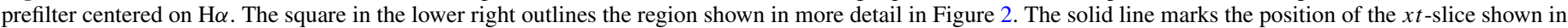
Figure 3. The letters a-g mark locations for the $\lambda t$-diagrams in Figure 6 . The arrows point in the direction of solar north and disk center.

(An animation of this figure is available in the online journal.)

the first time that sunspot chromospheres are ubiquitously filled with periodic jets that resemble dynamic fibrils in their physics but have significantly smaller extents.

\section{OBSERVATIONS AND DATA PROCESSING}

Two observational datasets obtained with the Swedish $1 \mathrm{~m}$ Solar Telescope (SST; Scharmer et al. 2003a) on La Palma are analyzed: one from the Solar Optical Universal Polarimeter (SOUP; Title \& Rosenberg 1981) and one from the CRisp Imaging SpectroPolarimeter (CRISP; Scharmer et al. 2008). The CRISP dataset is the main dataset and will be discussed first.

CRISP is a Fabry-Pérot tunable filter instrument that is capable of fast wavelength switching $(<50 \mathrm{~ms})$ so that densely sampled spectral profiles can be obtained in a relatively short time. The time series analyzed here was obtained on 2011 May 4 between 08:30 and 09:22 UT. The target sunspot (AR11204) was located at heliocentric coordinates $(x, y)=\left(-336^{\prime \prime}, 332^{\prime \prime}\right)$ and observing angle $\theta=30^{\circ}(\mu=0.87)$.

The CRISP observing program covered both the $\mathrm{H} \alpha$ and $\mathrm{Ca}$ II 8542 spectral lines. $\mathrm{H} \alpha$ was sampled at two line positions, at line center and in the red wing at $+1300 \mathrm{~mA}$. Ca II 8542 was sampled at 14 line positions in spectropolarimetric mode so that profiles were obtained for the four Stokes parameters $I, Q, U$, and $V$. Nine positions were sampled between $\Delta \lambda= \pm 300 \mathrm{~m} \AA$ at $75 \mathrm{~m} \AA$ steps, plus $\Delta \lambda= \pm 400, \pm 860$, and $+3100 \mathrm{~m} \AA$ from line center. The time to complete the acquisition of the two lines was less than $16 \mathrm{~s}$; the 52 minute time series comprises 193 time steps.

High spatial resolution close to the diffraction limit of the telescope was achieved with the aid of the adaptive optics system (Scharmer et al. 2003b) and image restoration using Multi-Object Multi-Frame Blind Deconvolution (van Noort et al. 2005). The processing of the CRISP data was based on the various procedures developed by Schnerr et al. (2011), Henriques (2012), van Noort \& Rouppe van der Voort (2008), and Selbing (2005). For a detailed description of the treatment of this dataset, in particular the polarimetric Ca II 8542 data, we refer the reader to de la Cruz Rodríguez et al. (2013b), who analyzed the same data in the context of umbral flashes.

After image restoration of the individual line scans and time steps, the time series were corrected for field rotation and residual offsets and rubber-sheet deformations, following Shine et al. (1994), using the wideband images as reference. The $\mathrm{H} \alpha$ time series was then aligned with the Ca II 8542 time series in order to account for small differences in the optical path of the two lines. The resulting effective field of view of the time series was $53^{\prime \prime} \times 52^{\prime \prime}$, with an image scale of $0{ }^{\prime} 059 \mathrm{pixel}^{-1}$.

The SOUP dataset was obtained on 2005 October 4 and was analyzed by De Pontieu et al. (2007a). The time series has a duration of 78 minutes and a $1 \mathrm{~s}$ cadence; it is centered on the $\mathrm{H} \alpha$ line core only. The field of view was centered on one of the larger spots (about 20" diameter) in AR10813 at $\mu=0.78$. A full characterization of the instrumentation and data is presented in De Pontieu et al. (2007a). For the remainder of the paper, we focus mainly on the CRISP dataset and we explicitly state when we analyze the SOUP data.

\subsection{Description of the Field of View}

One corner of the field of view is dominated by the large leading sunspot of AR11204; see Figure 1. The wideband image shows a sunspot with a double umbra that is separated by a strong light bridge. Some regions of the umbra have dense concentrations of umbral dots or light bridge-like structures. The umbra is partly surrounded by penumbra; a large section of the umbra that is facing the trailing, opposite polarity of the AR does not have penumbra. The small pore at $(X, Y)=\left(7^{\prime \prime}, 35^{\prime \prime}\right)$ 

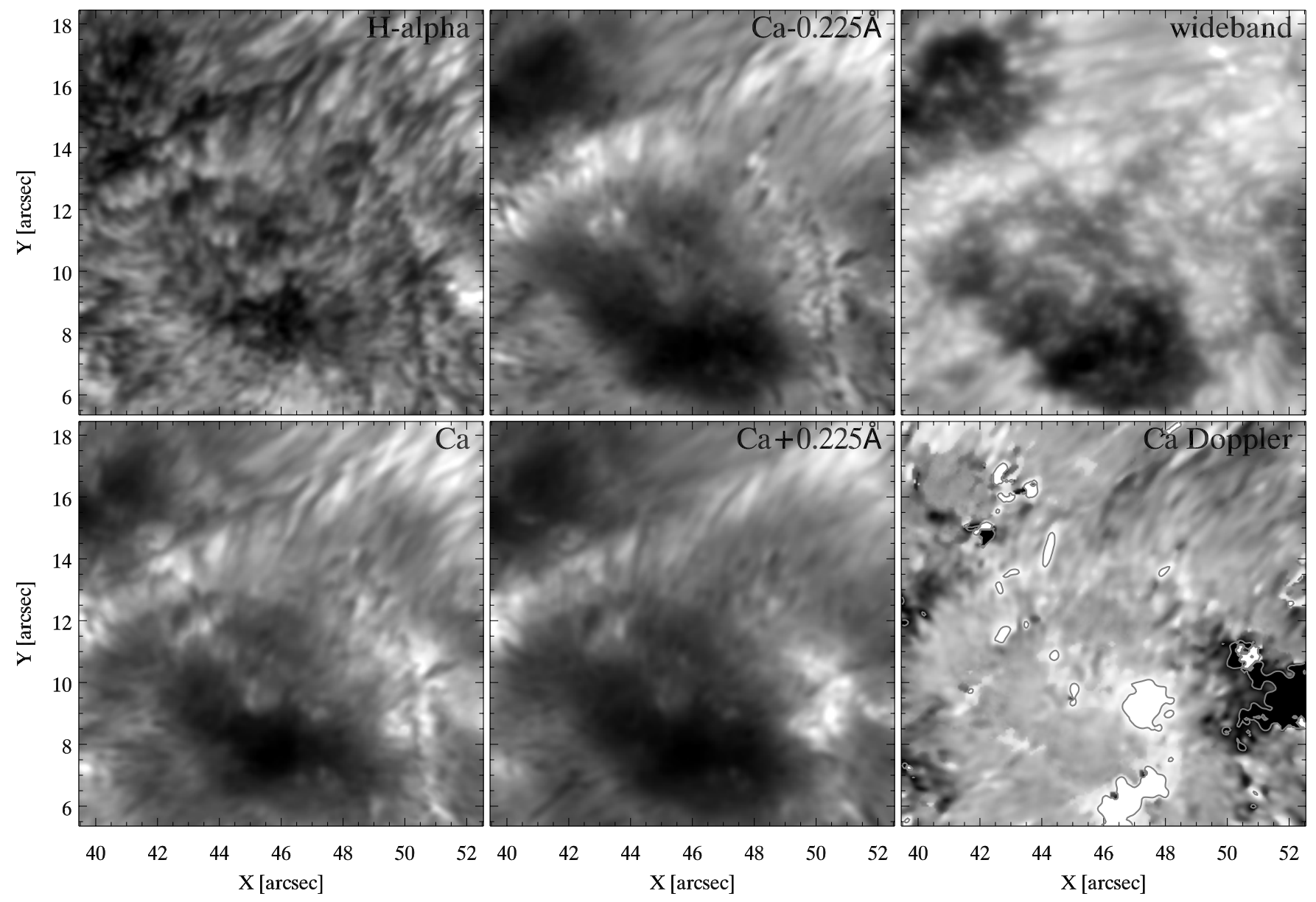

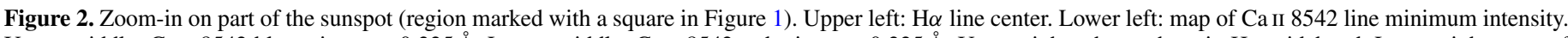

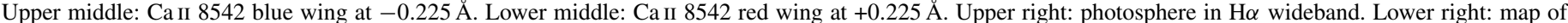
Ca II 8542 Doppler shift, scaled between $\pm 5 \mathrm{~km} \mathrm{~s}^{-1}$; the gray contours mark regions where the parabolic fit did not yield a reasonable Doppler measurement.

(An animation of this figure is available in the online journal.)

has opposite magnetic polarity and is at the front edge of the trailing plage region that is mostly outside of the field of view.

The $\mathrm{H} \alpha$ image in Figure 1 shows an AR chromosphere where the left half of the image is dominated by long $\left(>20^{\prime \prime}\right)$, apparently almost horizontal fibrils that connect the two polarities in the AR. Similarly long fibrils can be found in the upper right part of the sunspot and seem to be rooted in the outer part of the penumbra. Shorter fibrils are found outside the sunspot in the region at the lower left of the sunspot (outlined by $X=20^{\prime \prime}-35^{\prime \prime}$ and $\left.Y=0^{\prime \prime}-20^{\prime \prime}\right)$ and above the sunspot right of the middle of the image (outlined by $X=25^{\prime \prime}-40^{\prime \prime}$ and $Y=25^{\prime \prime}-52^{\prime \prime}$ ). These shorter fibrils are more dynamic than the longer fibrils and periodically rise and fall in a matter of minutes, as can be clearly seen in the $\mathrm{H} \alpha$ movie that is part of the online animation of Figure 1. These are dynamic fibrils; they were described in detail by Hansteen et al. (2006) and De Pontieu et al. (2007a). For the remainder of this paper, we refer to these two papers as VH06 and BDP07.

In the umbra, the $\mathrm{H} \alpha$ image shows a lot of fine detail in the form of dark specks against a brighter background. In the inner penumbra, the darker specks are more elongated. These darks specks are periodically varying in extension and linear position in the $\mathrm{H} \alpha$ movie and appear to be superposed on the large-scale pattern of intensity oscillations that is associated with the well-known chromospheric sunspot oscillations and running penumbral waves. Zooming in on the umbra and inner penumbra (best seen in the online animation associated with Figure 2), it is clear that the dark specks periodically move up and down on a smaller scale but in a similar way as the dynamic fibrils in the plage regions outside the sunspot. These small-scale sunspot dynamic fibrils are the main subject of the analysis in this paper.

\subsection{Measurement Methods}

The multi-dimensional CRISP dataset (with multiple diagnostics ( $\mathrm{H} \alpha$ and $\mathrm{Ca}$ II 8542) and associated wideband channels, spatial dimensions $X$ and $Y$, temporal dimension $t$, and spectral dimension $\lambda$ ) can be effectively explored with the Crisp Spectral Explorer (CRISPEX; Vissers \& Rouppe van der Voort 2012), a widget-based IDL tool that allows quick access to practically any cut through the dataset. CRISPEX offers videoplayer functionality for visualization of the time series of the two spectral lines side by side and functionality for zooming into regions of interest and quickly switching between spectral line positions. Quick inspection of the spectral evolution at different locations is made possible by real-time visualization of the $\lambda t$-diagram of the pixel under the mouse cursor. Space-time diagrams ( $x t$-diagrams) can be quickly extracted from the data along any spatial path (linear or curved) and the associated data can be stored separately for more detailed analysis. The versatile functionality of CRISPEX was an effective way to confirm 
the nature of the plage fibrils as dynamic fibrils in the context of the work by VH06 and BDP07 and to establish the similarity of the small-scale sunspot fibrils and the dynamic fibrils.

For measuring the physical properties of the sunspot fibrils, we follow the same methods as VH06, BDP07, Rouppe van der Voort et al. (2007), and Langangen et al. (2008b). $x t$ diagrams were extracted along linear paths aligned with the main axis of individual fibrils. Just as for dynamic fibrils in plage, the tops of the fibrils follow a path that is well approximated by a parabola. The parabolic fit to the top of fibril in the $x t$ diagram is then used to determine the velocity, deceleration, duration, and maximum height of the fibril.

To investigate the relationship between the magnetic field vector and the fibril properties, we inverted the Stokes Ca II 8542 data of a $34^{\prime \prime} \times 27^{\prime \prime}$ region to determine the magnetic field vectors. We used the spectropolarimetric inversion code Nicole (Socas-Navarro et al. 2000c) for LTE inversions of $286 \times$ 225 pixels (every second pixel along the $x$ and $y$ dimension). de la Cruz Rodríguez et al. (2012) suggested the reliability of LTE inversions for chromospheric magnetic field measurements, which we conveniently use in this work to retrieve the magnetic field vector, although Nicole is primarily a non-LTE inversion code.

More details on the inversions of the Ca II 8542 data can be found in de la Cruz Rodríguez et al. (2013b). In that study, LTE inversions were performed for the full time series of a small patch that covered the umbra and inner penumbra in a region with strong Stokes $Q$ and $U$ signal. It was shown that the magnetic field inclination for this patch was very stable throughout the time series, which justifies our generalization of using one time step as a measure of the inclination for the entire time series. The inversions provide information on the magnetic field vector at a height that is well above the level that is probed by standard photospheric polarimetric diagnostics. The peak in polarimetric sensitivity for the Ca II 8542 line is around $\pm 200 \mathrm{~m} \AA$, which probes the low chromosphere or an approximate height of $800 \mathrm{~km}$ in the quiet Sun (see de la Cruz Rodríguez et al. 2012).

\section{DYNAMIC FIBRILS IN THE SUNSPOT}

Figure 2 shows a zoom-in on part of the sunspot for different diagnostics. The $\mathrm{H} \alpha$ image is an image with CRISP tuned to the nominal line center. The $\mathrm{Ca}$ image below is a map of the minimum intensity in the line per pixel (so it is not the Ca II 8542 image of the CRISP nominal line center tuning). The reason for showing the line minimum intensity accounting for the wavelength shift is that the Ca II 8542 line is much more sensitive to Doppler shifts than $\mathrm{H} \alpha$ and the amplitude of the Doppler shifts associated with the dynamic fibril motion is large enough such that one cannot cover the full evolution of the fibril by just tuning to one fixed wavelength. An image of the Ca II 8542 line minimum intensity shows the dynamic fibrils much more clearly. The middle panels show the blue and red wing of Ca II 8542 at a corresponding Doppler offset of $8 \mathrm{~km} \mathrm{~s}^{-1}$; the Ca Doppler image is a map of the shift of the Ca II 8542 line measured from a parabolic fit to the central five line positions. This simple method is only relevant for simple absorption profiles, not for complicated line profiles with emission reversals, as, for example, umbral flashes (see de la Cruz Rodríguez et al. 2013b) or flat bottom ("raised core") profiles (de la Cruz Rodríguez et al. 2013a). The wideband image serves as photospheric reference.
While the small-scale fibrils are visible in the $\mathrm{H} \alpha$ and Ca II 8542 panels of Figure 2, we strongly encourage the reader to view the accompanying animation that shows the full time series for all panels. In the $\mathrm{H} \alpha$ and $\mathrm{Ca}$ movies, the periodic linear up and down motion of the small fibrils is very clear. Blinking the $\mathrm{H} \alpha$ and $\mathrm{Ca}$ II 8542 line minimum intensity images shows a close correspondence of the small fibrils in both diagnostics with a small offset of $\mathrm{H} \alpha$ being slightly taller, or higher, than $\mathrm{Ca}$, suggesting a slightly higher formation height for $\mathrm{H} \alpha$ than for $\mathrm{Ca}$ II 8542. A striking difference between the two diagnostics is that in $\mathrm{H} \alpha$, the pattern of small dark specks on a brighter background is visible everywhere in the sunspot. In $\mathrm{Ca}$ II 8542, on the other hand, there are patches dominated by strong intensity fluctuations caused by umbral flashes where the small fibrils are hardly or not at all discernible. The static $\mathrm{Ca}$ image in Figure 2 shows such a region as a dark patch between $X=44^{\prime \prime}-47^{\prime \prime}$ and $Y=6^{\prime \prime}-9^{\prime \prime}$. At this instant, the region is in a quiescent state between flashes.

The small fibrils are clearly visible in the blue and red wings in the middle panels of Figure 2 and the movie vividly displays the effect of Doppler shifts in this diagnostic: in the blue wing, the fibrils are exclusively visible as growing and up-moving (and therefore blueshifted), while in the red wing the dark fibrils are exclusively shrinking and down-moving. The Ca Doppler image shows both dark (blueshifted) and white (redshifted) patches and in the movie one can see both up-moving blueshifted (black) fibrils and down-moving redshifted (white) fibrils. The up-moving blueshifted fibrils appear to dominate the Doppler movie; this effect might be a visual impression (the eye is possibly more susceptible to moving dark features) combined with the possibility that the simple method of measuring the Doppler shift from a parabolic fit to the line is less effective for the down-moving phase.

The typical width of the fibrils is found to be around 0.3 $(200 \mathrm{~km})$. We sometimes see faint examples with widths down to the diffraction limit of the telescope $(\lambda / D=0$ ' 14 at $\lambda=656.3$ $\mathrm{nm})$. Wider examples of the order of 0.5 sometimes give the impression of sub-structure as if the fibrils are in closely aligned pairs.

The parabolic nature of the trajectory of the top of the dynamic fibrils in the sunspot is clearly seen in Figure 3. An $x t$ diagram is shown for a linear path that crosses from the umbra through the penumbra and into the surrounding plage (see the location of the path marked in Figure 1). The different extensions of the parabolas depending on the position along the path or location in the sunspot is striking: from very small in the umbra in the bottom of the diagram, to progressively larger parabolas in the outer penumbra and beyond the sunspot. The umbral parabolas also have a clearly shorter duration than the parabolas in the outer penumbra and the surrounding plage. Another notable regional difference in the $x t$ diagram is the regularity of the appearance of parabolas in the umbra and inner penumbra and the more irregular appearance of parabolas further out. In the region $x \sim 2-3 \mathrm{Mm}$, different parabolas appear progressively later or with a phase delay. This result is particularly clear for $t=1900-2500 \mathrm{~s}$. This part of the diagram crosses the inner penumbra where running penumbral waves are observed.

The sunspot dynamic fibrils were highly resolved in the temporal domain in the $1 \mathrm{~s}$ cadence SOUP dataset. Various aspects of the $x t$ diagram discussed above are also prominent in the SOUP $x t$ diagram in Figure 4, including the parabolic trajectories of the dynamic fibril tops, varying extensions (low in the umbra, progressively larger in the outer umbra and 


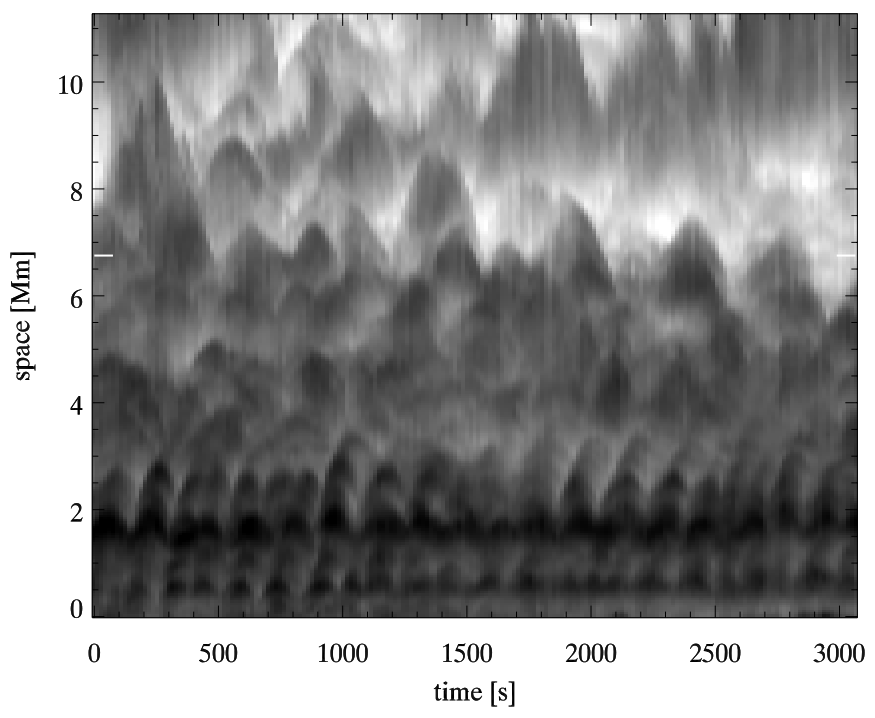

Figure 3. $\mathrm{H} \alpha x t$ diagram along a line that starts in the umbra (at 0 ) and crosses through the penumbra parallel to the penumbral filaments (see the line marked in Figure 1). The (photospheric) outer penumbral boundary is at $6.8 \mathrm{Mm}$ and is marked with a thin white line.

penumbra), and the regularity in the recurring dynamic fibrils. In the region $x \sim 3-5 \mathrm{Mm}$, we observe a similar phase delay in the occurrence of dynamic fibrils as in Figure 3.

Detailed $x t$ diagrams for three different dynamic fibrils are shown for various diagnostics in Figure 5: the $\mathrm{H} \alpha$ line core, the Ca II 8542 minimum intensity, and five different line positions in Ca II 8542. The shortest (both in length and duration) dynamic fibril is in the umbra (top row), the dynamic fibril in the light bridge is slightly taller and has a longer duration (middle row), and the penumbral dynamic fibril is tallest (bottom row). From a comparison of the left two columns (and the fifth column " $\mathrm{Ca}$ $+0.000 \AA$ " at nominal line center), we see that the dynamic fibril in $\mathrm{H} \alpha$ is slightly above $\mathrm{Ca}$ II 8542 . It is interesting to note how the different phases in the life of a dynamic fibril can be traced through the wings of $\mathrm{Ca}$ II 8542 : the dynamic fibril first appears in the blue wing at $-0.400 \AA$ and $-0.225 \AA$ and disappears again in the blue wing when it reaches maximum height at line center. After maximum height, the down-moving phase can be followed in the red wing positions.

The spectral evolution at different locations in the sunspot is shown in Figure 6. The spectral signature of dynamic fibrils is a diagonal line starting from maximum blueshift at the start of the event with constant negative acceleration through $0 \mathrm{~km} \mathrm{~s}^{-1}$ to maximum redshift before disappearance. Langangen et al. (2008a) showed similar spectral signatures for dynamic fibrils in Ca II 8662 in their Figure 2. All $\lambda t$ diagrams show these signatures except panel $a$, which is from the dark umbral core dominated by umbral flashes. The regular appearance of the central reversals during the flashes is clearly visible. Note that in the $\mathrm{H} \alpha$ line center images, the small dynamic fibrils seem to be present in this region along with $\mathrm{Ca}$ II 8542 umbral flashes and an absence of dynamic fibril spectral signatures. The amplitude of the dynamic fibril excursions is on the order of $\pm 10 \mathrm{~km} \mathrm{~s}^{-1}$. Note the small amplitudes in panel $(\mathrm{g})$, which is located in the penumbra in a region where the filaments are almost parallel to the disk center direction. This region is where there is the strongest signal in linear polarisation in Stokes $Q$ and $U$ and the magnetic field is almost perpendicular to the line of sight. The plasma motion in the dynamic fibril is almost

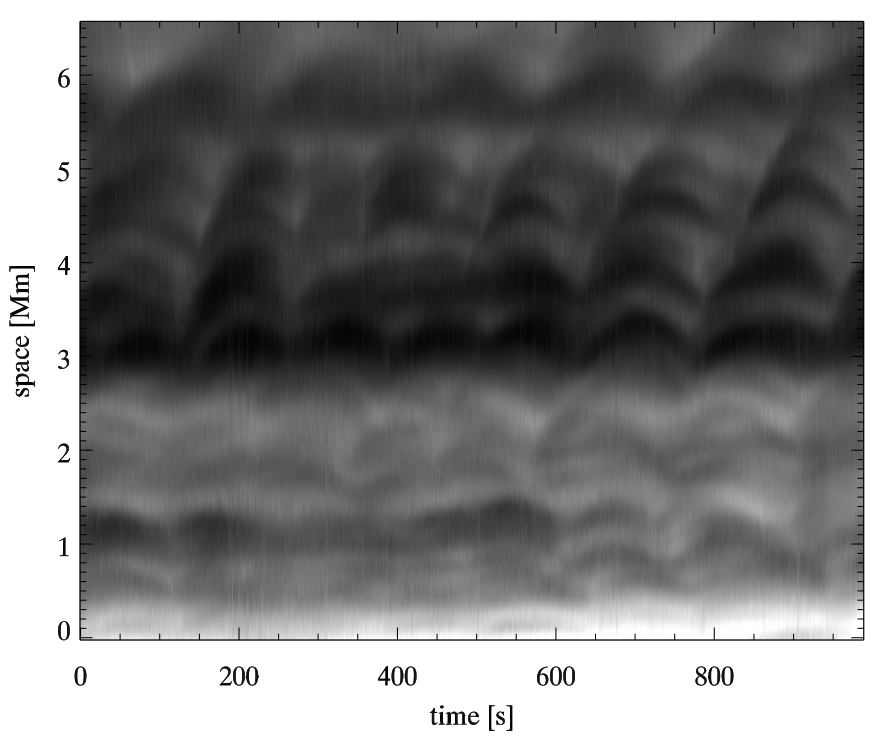

Figure 4. H $\alpha x t$ diagram from the 2005 October 4 SOUP data with a 1 s cadence. The slit covered the umbra almost completely (at the bottom of the diagram), the full (but narrow) penumbra, and a small piece of the surroundings (from about $5.5 \mathrm{Mm})$.

perpendicular to the line of sight in this region and we therefore observe only small amplitudes in the $\lambda t$ diagram.

A total of 460 dynamic fibrils were measured by fitting parabolas to the path of their top in the $x t$ diagrams. Maximum velocity, deceleration, duration, and maximum length valves are presented in scatter plots in Figure 7. BDP07 demonstrated that there is a clear correlation between both maximum velocity and deceleration and duration and maximum length of the dynamic fibrils in plage. We show that dynamic fibrils in a sunspot show the same correlations; the data points of BDP07 are added for comparison. Note that our data have a significantly lower temporal resolution than the data of BDP07 (almost 16 times lower). This fact explains the concentration of the duration measurements in our data in discrete bins that are integer multiples of the temporal cadence.

The location of the 460 analyzed dynamic fibrils in the sunspot is illustrated by colored lines in the right panel of Figure 8 . The length of the lines indicates the maximum extension of the top of the dynamic fibrils; the color is a measure of the duration. These properties can be compared with the inclination of the local magnetic field, which can be inferred from the left panel. Despite the noise in the map, the general picture of the inclination in the lower chromosphere is that of almost vertical fields in the umbra and progressively more horizontal fields in the penumbra at increasing distance from the umbra. This result conforms with the paradigm of the general magnetic field topology in sunspots. The reliability of the magnetic field inclination measurements is obviously correlated with the signal in Stokes parameters $V, Q$, and $U$. The strongest (relative) signals, particularly in linear polarisation, are found in the limb-side umbra and penumbra (cf. Figure 2 in de la Cruz Rodríguez et al. 2013b) and the dynamic fibrils are preferentially measured in these regions. From the comparison between the two panels in Figure 8, a clear trend emerges for spatially longer, long-period dynamic fibrils to be located in regions with more horizontal magnetic fields.

These correlations of duration versus magnetic field inclination angle and length versus inclination angle are clear from the scatter plots in the bottom panels of Figure 8 . The values of the 

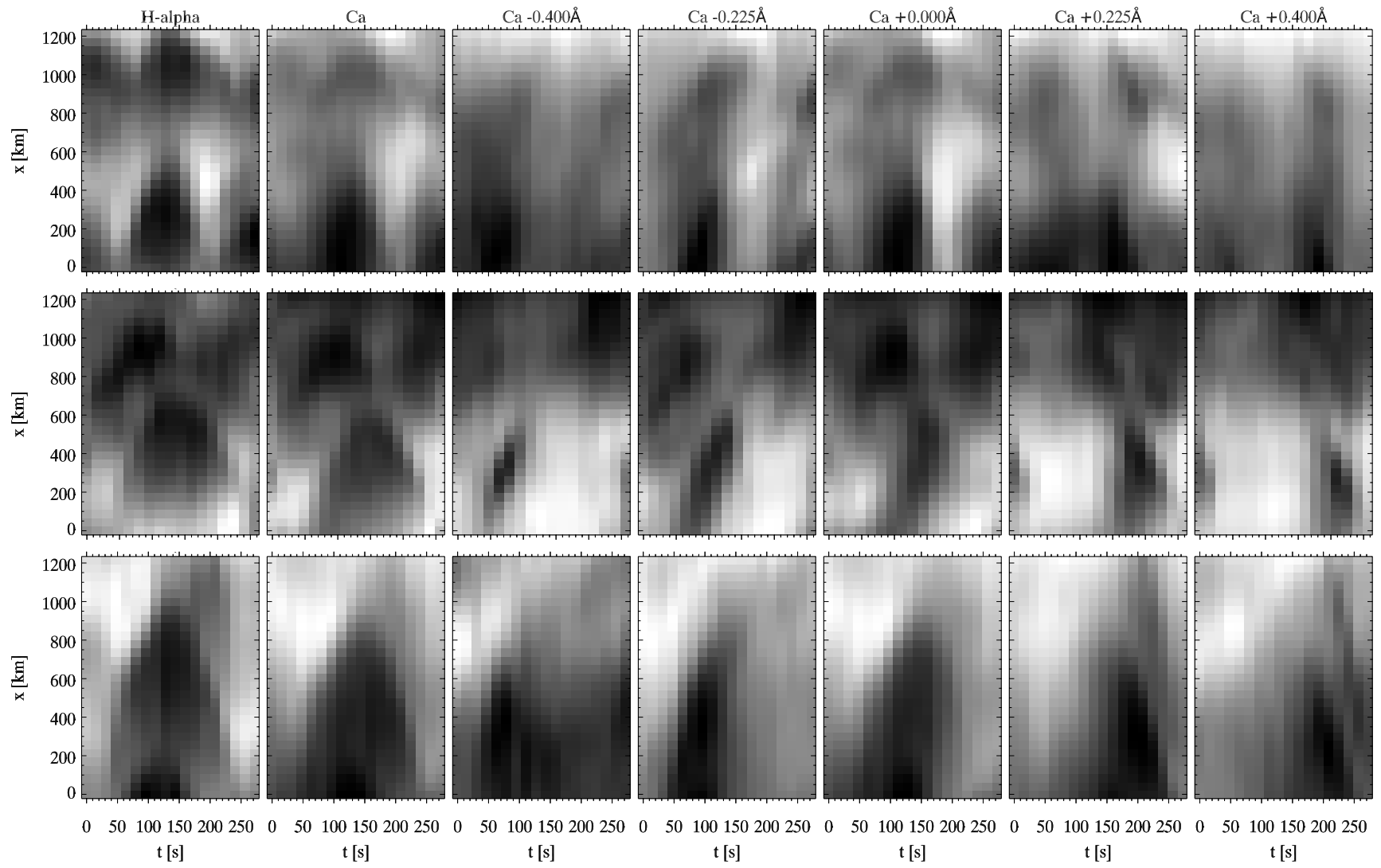

$\mathrm{t}[\mathrm{s}]$

$\mathrm{t}[\mathrm{s}]$

$\mathrm{t}[\mathrm{s}] \quad \mathrm{t}[\mathrm{s}]$

Figure 5. $x t$ diagrams for three different dynamic fibrils in various diagnostics marked at the top of the columns. Top row: umbral dynamic fibril, middle row: dynamic fibril in light bridge, bottom row: penumbral dynamic fibril. The column marked "Ca" is the Ca II 8542 line minimum intensity; "Ca $+0.000 \AA$ " is at the nominal line center at $8542 \AA$.
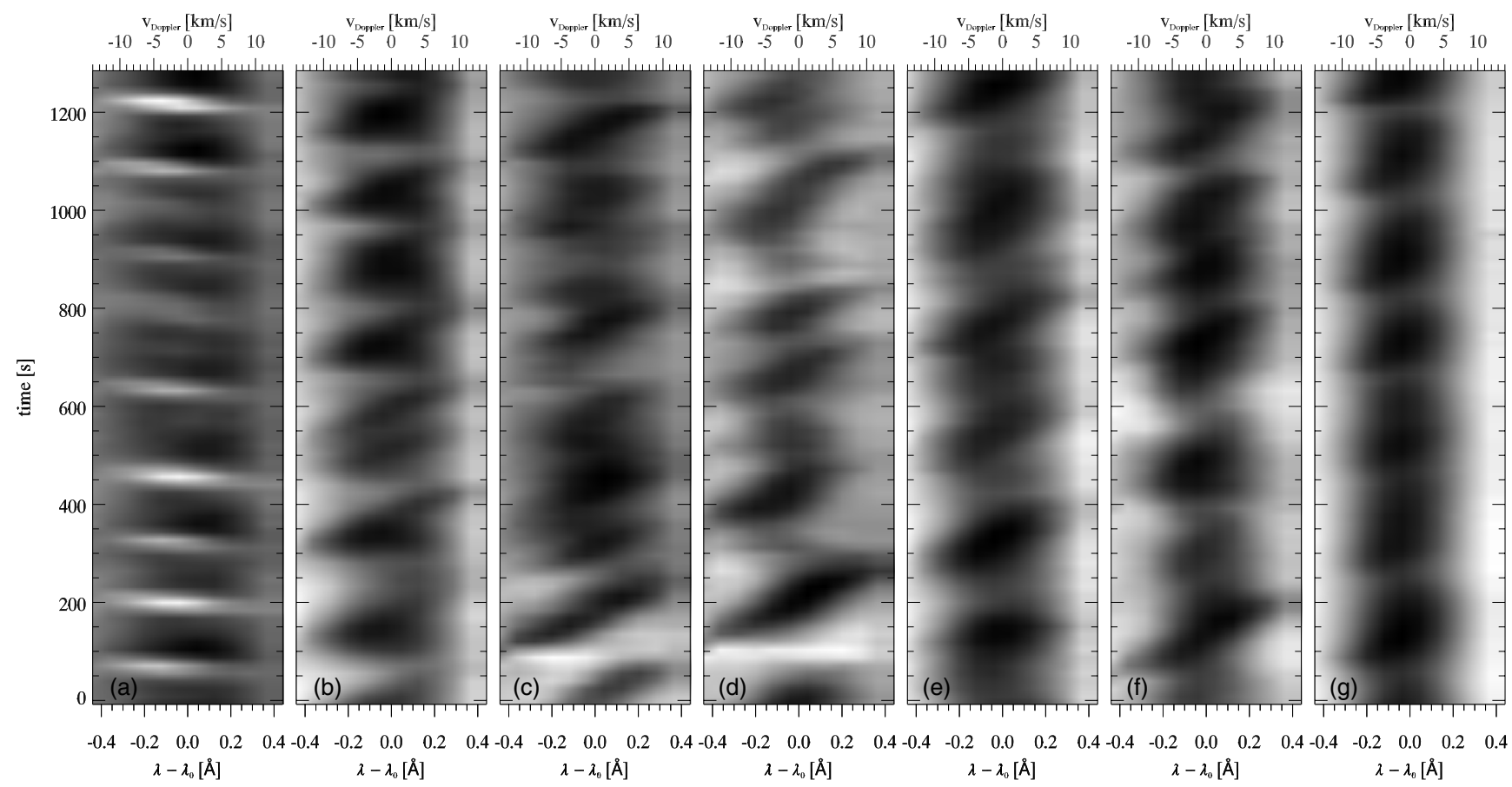

$\lambda-\lambda_{0}[\AA ̊ \AA]$

$\left.\lambda-\lambda_{0}[\AA]\right]$

$\lambda-\lambda_{0}[\AA ̊]$

$\left.\lambda-\lambda_{0}[\AA]\right]$

$\lambda-\lambda_{0}[\AA]$

$\begin{array}{lllll}-0.2 & 0.0 & 0.2 & 0.4\end{array}$

Figure 6. Ca II $8542 \lambda t$ diagrams for various locations in the sunspot. (a) In the dark umbral core, (b) in the umbra in areas with umbral dots, (c) in the umbra in areas with large umbral dots/light bridge like structures, (d) in the umbra close to the light bridge structure, (e) in the strong light bridge, (f) in the amorphous penumbral area, $(\mathrm{g})$ in the penumbra.

field inclination angle are averages over $3 \times 3$ pixels centered on the lowest point of the path of the top of the dynamic fibril. As discussed above, the low temporal cadence of the data results in concentration of the duration values in discrete bins, making it difficult to differentiate between data points in high-density areas in the left scatter plot. Therefore, a smoothed density map of the data points is shown in the background so that the visual trend in the data is better illustrated. 

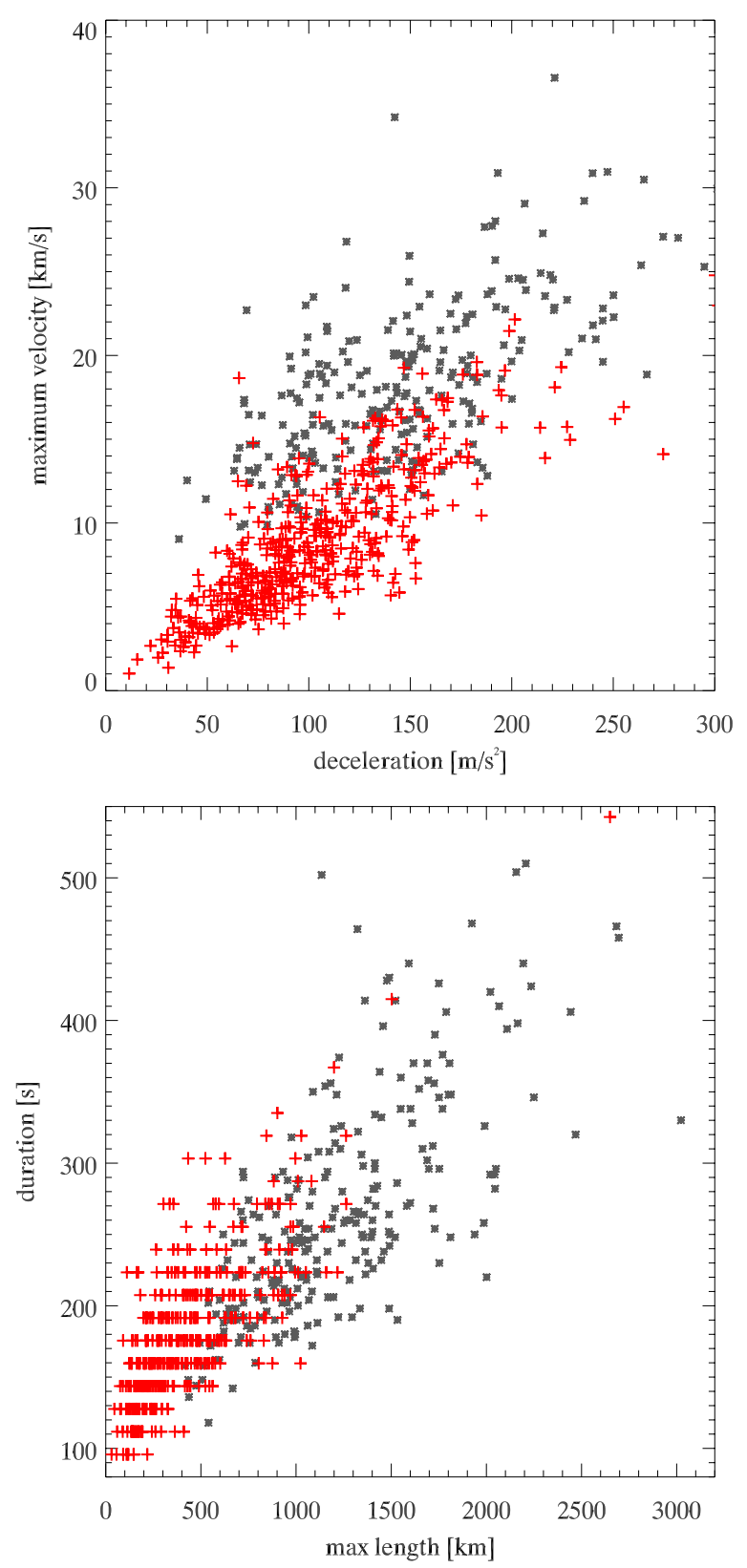

Figure 7. Scatter plots of dynamic fibril properties. The red crosses are the measurements in the sunspot (460 individual dynamic fibrils); the smaller gray asterisks are dynamic fibril properties from BDP07.

(A color version of this figure is available in the online journal.)

\section{DISCUSSION AND CONCLUSIONS}

Our new high-resolution SST observations reveal the ubiquitous presence of periodic, small-extent jets in the chromosphere of sunspots. These jets follow parabolic trajectories in the space-time domain and appear to be the sunspot counterpart of the much longer dynamic fibrils found in plage regions, for which the physics is well established (VH06; BDP07; Heggland et al. 2007). We find a strong correlation between both maximum velocity and deceleration and duration and maximum length for the tops of a sample of 460 sunspot dynamic fibrils. These correlations agree well with corresponding measurements of dynamic fibrils in plage (VH06; BDP07; Langangen et al. 2008a, 2008b) and some mottles in the quiet Sun (Rouppe van der Voort et al. 2007). In these studies, numerical simulations were used to iden- tify these correlations as signatures that these periodic jets are driven by waves that propagate from the lower atmosphere into the chromosphere where they steepen into shocks. The sunspot dynamic fibrils have generally shorter maximum lengths, shorter durations, lower maximum velocities, and smaller declerations than the dynamic fibrils in plage. However, given the convincing similarities, we conclude that their driving mechanism and nature must be essentially the same. Therefore, this new solar feature represents a new class within this family of dynamic field-guided wave phenomena.

The more idealized (and therefore controlled) numerical simulations by Heggland et al. (2007) showed that there is a linear correlation between maximum velocity and deceleration for shock wave-driven chromospheric jets. The slope of the correlation is primarily determined by the period of the waves. Since the period of the wave train remains constant during the propagation and steepening into shock waves, there is a given time during which the amplitude can change between extrema. So, for fixed period waves of varying maximum velocity, higher amplitude velocities will be subject to larger decelerations. For varying periods, the longer period waves will experience smaller decelerations. These experiments suggest that the observed spread in the scatter plots of Figure 7 can be partly attributed to variation in the excitation periods. The spread in the scatter plot of duration versus maximum length is also introduced by projection effects in the measurement of the length. From the map of magnetic field inclination angle in Figure 8, it is clear that the viewing angles of dynamic fibrils in different areas in the sunspot vary significantly. We do not attempt to correct for these projection effects but note that this effect introduces a spread into the length measurements. Obvious alternative major contributors to the spread are various errors in the measurements and the observations.

The simulations of Heggland et al. (2007) and later by Heggland et al. (2011) elaborated on the idea that long-period waves can propagate along inclined magnetic fields into higher and lower density regions of the solar atmosphere (De Pontieu et al. 2004; and earlier investigated by Michalitsanos 1973; Bel \& Leroy 1977; Suematsu 1990). Inclined magnetic fields alter the acoustic cutoff period for waves that move along the magnetic field due to a reduced effective gravity so that long-period waves propagate into higher atmospheric regions where they otherwise would be evanescent in non-magnetic regions. From our observations, we can directly compare the inclination of the magnetic field (converted to the local frame on the Sun as in Scharmer et al. 2013) and the period of the sunspot dynamic fibrils (Figure 8). Indeed, we find a clear trend that the longer duration dynamic fibrils are found in regions with more inclined magnetic fields. This result provides direct observational support that this mechanism is at work at driving dynamic fibrils. VH06 and BDP07 found indirect support from significant differences in the dynamic fibril characteristics for two different regions where crude magnetic field extrapolations of low-resolution MDI magnetic field maps indicated regional differences in the general magnetic field inclination. For our data, we can determine the local magnetic field vector from LTE inversions of the Stokes Ca II 8542 data and measure the magnetic field inclination at approximately the same height as the dynamic fibrils. On much larger scales, the modification of the acoustic cutoff frequency by the change in the magnetic field inclination was demonstrated for a sunspot (McIntosh \& Jefferies 2006) and supergranular boundaries (Jefferies et al. 2006). 

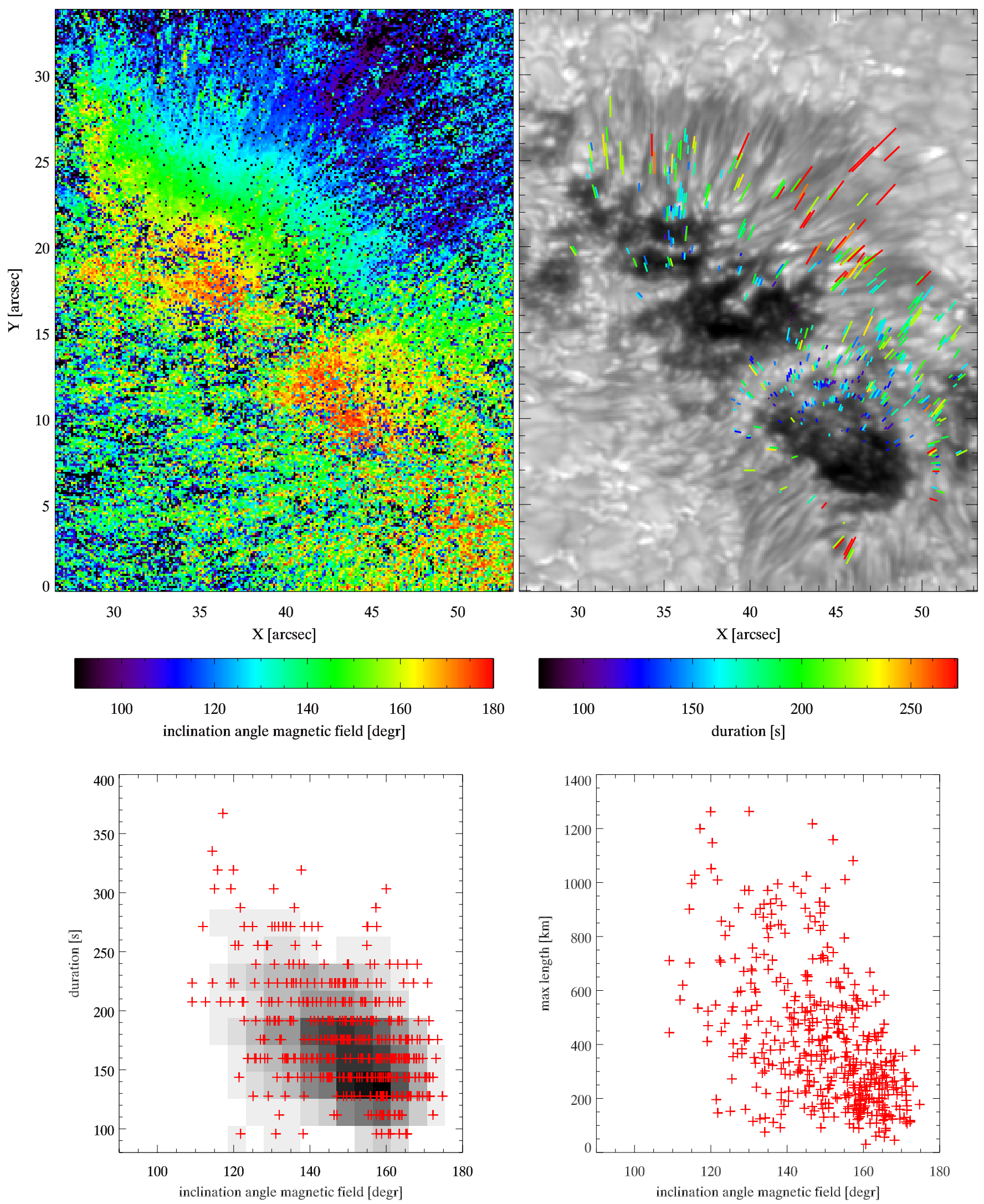

Figure 8. Relation between magnetic field inclination and dynamic fibril duration and length. The top left panel shows a map of the magnetic field inclination from LTE inversion of the Ca II 8542 Stokes data. $180^{\circ}$ is a vertical, negative polarity field; $90^{\circ}$ is a horizontal field. The top right panel shows a continuum Ca II 8542 image with the paths of the top of 460 dynamic fibrils drawn. The color of the paths indicates the duration of the dynamic fibril. The bottom left panel shows a scatter plot of the duration of the dynamic fibrils vs. the inclination angle of the magnetic field at the lowest point of the path of the top of the dynamic fibril (red crosses). The inclination angle is the average value of a $3 \times 3$ pixel area. In the background, a smoothed density image of the same data points is shown with an inverted color table. The bottom right panel shows a scatter plot of the maximum length of the path of the top of the dynamic fibrils vs. the magnetic field inclination.

(A color version of this figure is available in the online journal.) 
While we determine the magnetic field inclination at approximately the same height as the dynamic fibrils, it is the field inclination at lower heights that is important for the evanescence or propagation of waves to chromospheric heights. If there is significant variation of the inclination with height, this fact would introduce spread into the scatter plots of Figure 8 and would reduce the correlation between the parameters. A possible way to study this effect would be to add photospheric spectropolarimetric diagnostics to the observing program, but we remark that it would be extremely difficult to achieve sufficient resolution in the vertical domain (i.e., height in the sunspot atmosphere) in order to study this effect in high detail.

Apart from the relation between magnetic field inclination and period of the dynamic fibrils, the right panel of Figure 8 suggests a positive correlation between dynamic fibril length and duration. A similar correlation was found for plage dynamic fibrils (BDP07) and in the MHD simulations of Martínez-Sykora et al. (2009) and Heggland et al. (2011).

The presence of upward propagating waves and shocks in sunspot atmospheres has been known from both observations (see, e.g., Lites 1992) and simulations (see, e.g., Bard \& Carlsson 2010). What is new from our high-resolution SST observations is the high degree of fine structure in the form of small specks or fibrils that seems to be superposed on the large-scale oscillatory pattern (i.e., the umbral flashes in Ca II 8542). It is possible that, there exist small-scale density fluctuations in the sunspot atmosphere resulting in local opacity differences that give rise to the apparent ejection of small-scale jets that are excited by larger scale wave fronts. A high degree of fine-scale structuring in the sunspot photosphere has been known for a long time to exist in the form of, for example, umbral dots, light bridges, and penumbral filaments (for reviews see, e.g., Solanki 2003; Thomas \& Weiss 2004). The highest resolution sunspot observations suggest photospheric substructure down to the diffraction limit of present-day telescopes (see, e.g., Scharmer et al. 2002; Lites et al. 2004; Ichimoto et al. 2007; Henriques 2013; Henriques \& Kiselman 2013). Recent simulation efforts explain the various fine-scale structures in sunspots as different manifestations of overturning convection in the strong magnetic field regime (see, e.g., Schüssler \& Vögler 2006; Heinemann et al. 2007; Rempel 2011). It is likely that such fine-scale structuring in the photosphere extends to higher layers and also results in small-scale lateral variations in the vertical propagation of waves.

The two-dimensional simulations of Heggland et al. (2011) showed that fine-scale structuring of chromospheric jets comes about naturally from slight differences in thermodynamic properties along neighboring field lines. Slight lateral differences lead to differences in shock speeds, and thus the formation of a highly corrugated shock front surface. The simulations show that initially smoothly and quasi-spherically expanding shock fronts nevertheless lead to skinny fingers of cold plasma protruding into the corona. This result seems to be a consequence of the fact that the path taken by the waves is not equally long for neighboring field lines and that the speed of sound varies for each field line. Also, this process depends on the history of the plasma, i.e., the passage of previous waves (which lead to varying temperature structuring). These complexities explain why the fine structuring we see in the sunspot chromosphere in $\mathrm{H} \alpha$ and $\mathrm{Ca}$ II 8542 is not a simple mapping of the photospheric structuring (in the form of umbral dots, light bridges, and penumbral fine structure) but is also a result of factors that affect the wave propagation above the photosphere such as slight lateral thermodynamic differences and the local wave history.

It is plausible that the fine-scale structuring in Ca II umbral flashes reported earlier from polarimetric inversions (SocasNavarro et al. 2000a, 2000b) and direct imaging (Socas-Navarro et al. 2009; Henriques \& Kiselman 2013) is related to the smallscale sunspot dynamic fibrils we report here. It is, however, likely that they are different from the more transient micro-jets observed in Ca II H in the penumbra (Katsukawa et al. 2007) and umbra (Bharti et al. 2013). These sunspot micro-jets appear to be a kind of jet that occurs much more infrequently, has shorter lifetime, is associated with brightening, and appears to be only rising. The penumbral micro-jets have been associated with magnetic reconnection processes (for example by Ryutova et al. 2008) and one might speculate that these could be the sunspot counterpart of the type II spicules at the limb. Following that analogy, the sunspot dynamic fibrils we report here would be the sunspot counterparts of the wave-driven type I spicules.

We finish by speculating on the general behavior of dynamic fibrils in different environments. There are significant differences in maximum lengths if one compares dynamic fibrils in sunspots, plage, the quiet Sun, and coronal holes. The longest dynamic fibrils are found in AR plage (BDP07). In sunspots, we observe a range of different lengths, with the shortest in the umbra and progressively longer ones away from the umbral center and into the penumbra but all typically shorter than in plage. The quiet Sun equivalent of AR dynamic fibrils, or mottles, are also typically shorter than in plage (Rouppe van der Voort et al. 2007). At the limb, dynamic fibrils are observed as type I spicules and in the extensive study of Pereira et al. (2012) only a very low number of parabolic spicules were found in the quiet Sun and coronal holes, typically less than $3 \%$ of the detected spicules; these features are completely absent in some datasets. This apparent low abundance of type I spicules is probably caused by these structures being short and therefore hidden in the dense forrest of spicules at the limb. In ARs, on the other hand, the majority of detected spicules are type I and reach maximum lengths that are similar to type II spicules in ARs, the quiet Sun, and coronal holes. The length variations in different regions are probably the result of a combination of factors: the input power or strength of the waves, the inclination angle of the magnetic field, and the spatial expansion of the magnetic field. In the quiet Sun and coronal holes, there is relatively large wave power but significant field expansion from the photosphere to the chromosphere, which leads to the shock wave energy being spread out over a large area, resulting in only short fibrils. In sunspots, the wave power is much lower and there is relatively low field expansion. For the umbra, the combination of low wave power and almost vertical field leads to very short dynamic fibrils. In addition, the magnetic field strength is strongest in the umbra, leading to lower gas density and lower shock height. In the penumbra, the fields get more inclined, leading to longer dynamic fibrils. There is probably relatively more convective input in the penumbra that leads to higher wave power and also contributes to longer dynamic fibrils. Finally, these factors conspire to create the longest dynamic fibrils in plage: there is more convective energy input than in sunspots, inclined fields enhance leakage of wave power into the chromosphere, and there is not as much field expansion as in the quiet Sun and coronal holes.

The authors thank Bart de Pontieu, Viggo Hansteen, Mats Carlsson, and Rob Rutten for fruitful discussions. The Swedish $1 \mathrm{~m}$ Solar Telescope was operated by the Institute for Solar 
Physics of the Royal Swedish Academy of Sciences in the Spanish Observatorio del Roque de los Muchachos of the Instituto de Astrofísica de Canarias. The authors gratefully acknowledge support from the International Space Science Institute (ISSI). Part of the computations were performed on resources provided by the Swedish National Infrastructure for Computing (SNIC) at Chalmers Centre for Computational Science and Engineering (C3SE) with project number SNIC002-12-27. This research was supported by the Research Council of Norway and by the European Research Council under the European Union's Seventh Framework Programme (FP7/2007-2013)/ERC grant agreement No. 291058. This research has made use of NASA's Astrophysics Data System.

\section{REFERENCES}

Bard, S., \& Carlsson, M. 2010, ApJ, 722, 888

Bel, N., \& Leroy, B. 1977, A\&A, 55, 239

Bharti, L., Hirzberger, J., \& Solanki, S. K. 2013, A\&A, 552, L1

de la Cruz Rodríguez, J., De Pontieu, B., Carlsson, M., \& Rouppe van der Voort, L. H. M. 2013a, ApJL, 764, L11

de la Cruz Rodríguez, J., Rouppe van der Voort, L., Socas-Navarro, H., \& van Noort, M. 2013b, A\&A, 556, A115

de la Cruz Rodríguez, J., Socas-Navarro, H., Carlsson, M., \& Leenaarts, J. 2012, A\&A, 543, A34

De Pontieu, B., Erdélyi, R., \& James, S. P. 2004, Natur, 430, 536

De Pontieu, B., Hansteen, V. H., Rouppe van der Voort, L., van Noort, M., \& Carlsson, M. 2007a, ApJ, 655, 624 (BDP07)

De Pontieu, B., McIntosh, S., Hansteen, V. H., et al. 2007b, PASJ, 59, 655

Hansteen, V. H., De Pontieu, B., Rouppe van der Voort, L., van Noort, M., \& Carlsson, M. 2006, ApJL, 647, L73 (VH06)

Heggland, L., De Pontieu, B., \& Hansteen, V. H. 2007, ApJ, 666, 1277

Heggland, L., Hansteen, V. H., De Pontieu, B., \& Carlsson, M. 2011, ApJ, 743,142

Heinemann, T., Nordlund, Å., Scharmer, G. B., \& Spruit, H. C. 2007, ApJ, 669,1390

Henriques, V. M. J. 2012, A\&A, 548, A114

Henriques, V. M. J. 2013, PhD thesis, Stockholm Univ., http://urn.kb.se/ resolve?urn=urn:nbn:se:su:diva- 86798

Henriques, V. M. J., \& Kiselman, D. 2013, A\&A, 557, A5

Ichimoto, K., Suematsu, Y., Tsuneta, S., et al. 2007, Sci, 318, 1597

Jefferies, S. M., McIntosh, S. W., Armstrong, J. D., et al. 2006, ApJL, 648, L151

Katsukawa, Y., Berger, T. E., Ichimoto, K., et al. 2007, Sci, 318, 1594

Langangen, Ø., Carlsson, M., Rouppe van der Voort, L., Hansteen, V., \& De Pontieu, B. 2008a, ApJ, 673, 1194
Langangen, Ø., Rouppe van der Voort, L., \& Lin, Y. 2008b, ApJ, 673, 1201

Lites, B. W. 1992, in NATO ASIC Proc. 375: Sunspots. Theory and Observations, ed. J. H. Thomas \& N. O. Weiss (Dordrecht: Kluwer), 261

Lites, B. W., Scharmer, G. B., Berger, T. E., \& Title, A. M. 2004, SoPh, 221,65

Martínez-Sykora, J., Hansteen, V., De Pontieu, B., \& Carlsson, M. 2009, ApJ, 701,1569

McIntosh, S. W., \& Jefferies, S. M. 2006, ApJL, 647, L77

Michalitsanos, A. G. 1973, SoPh, 30, 47

Nagashima, K., Sekii, T., Kosovichev, A. G., et al. 2007, PASJ, 59, 631

Pereira, T. M. D., De Pontieu, B., \& Carlsson, M. 2012, ApJ, 759, 18

Rempel, M. 2011, ApJ, 729, 5

Rouppe van der Voort, L. H. M., De Pontieu, B., Hansteen, V. H., Carlsson, M., \& van Noort, M. 2007, ApJL, 660, L169

Rouppe van der Voort, L. H. M., Rutten, R. J., Sütterlin, P., Sloover, P. J., \& Krijger, J. M. 2003, A\&A, 403, 277

Ryutova, M., Berger, T., Frank, Z., \& Title, A. 2008, ApJ, 686, 1404

Scharmer, G. B., Bjelksjö, K., Korhonen, T. K., Lindberg, B., \& Petterson, B. 2003a, Proc. SPIE, 4853, 341

Scharmer, G. B., de la Cruz Rodriguez, J., Sütterlin, P., \& Henriques, V. M. J. 2013, A\&A, 553, A63

Scharmer, G. B., Dettori, P. M., Lofdahl, M. G., \& Shand, M. 2003b, Proc. SPIE, 4853, 370

Scharmer, G. B., Gudiksen, B. V., Kiselman, D., Löfdahl, M. G., \& Rouppe van der Voort, L. H. M. 2002, Natur, 420, 151

Scharmer, G. B., Narayan, G., Hillberg, T., et al. 2008, ApJL, 689, L69

Schnerr, R. S., de La Cruz Rodríguez, J., \& van Noort, M. 2011, A\&A, 534, A45

Schüssler, M., \& Vögler, A. 2006, ApJL, 641, L73

Selbing, J. 2005, MSc thesis, Stockholm University

Shibata, K., \& Suematsu, Y. 1982, SoPh, 78, 333

Shine, R. A., Title, A. M., Tarbell, T. D., et al. 1994, ApJ, 430, 413

Socas-Navarro, H., McIntosh, S. W., Centeno, R., de Wijn, A. G., \& Lites, B. W 2009, ApJ, 696, 1683

Socas-Navarro, H., Trujillo Bueno, J., \& Ruiz Cobo, B. 2000a, ApJ, 544, 1141

Socas-Navarro, H., Trujillo Bueno, J., \& Ruiz Cobo, B. 2000b, Sci, 288, 1396

Socas-Navarro, H., Trujillo Bueno, J., \& Ruiz Cobo, B. 2000c, ApJ, 530, 977

Solanki, S. K. 2003, A\&ARv, 11, 153

Suematsu, Y. 1990, in Progress of Seismology of the Sun and Stars, ed. Y. Osaki \& H. Shibahashi (Lecture Notes in Physics, Vol. 367; Berlin: Springer), 211

Suematsu, Y., Shibata, K., Neshikawa, T., \& Kitai, R. 1982, SoPh, 75, 99

Thomas, J. H., \& Weiss, N. O. 2004, ARA\&A, 42, 517

Title, A., \& Rosenberg, W. 1981, OptEn, 20, 815

Tsiropoula, G., Tziotziou, K., Kontogiannis, I., et al. 2012, SSRv, 169, 181

van Noort, M., Rouppe van der Voort, L., \& Löfdahl, M. G. 2005, SoPh, 228,191

van Noort, M. J., \& Rouppe van der Voort, L. H. M. 2008, A\&A, 489, 429

Vissers, G., \& Rouppe van der Voort, L. 2012, ApJ, 750, 22 